\title{
NMR study of xenotropic murine leukemia virus-related virus protease in a complex with amprenavir.
}

\section{$\operatorname{AUTHOR}(\mathrm{S}):$}

Furukawa, Ayako; Okamura, Hideyasu; Morishita, Ryo; Matsunaga, Satoko; Kobayashi, Naohiro; Ikegami, Takahisa; Kodaki, Tsutomu; ... Ryo, Akihide; Nagata, Takashi; Katahira, Masato

\section{CITATION:}

Furukawa, Ayako ...[et al]. NMR study of xenotropic murine leukemia virus-related virus protease in a complex with amprenavir.. Biochemical and biophysical research communications 2012, 425(2): 284-289

\section{ISSUE DATE:}

2012-08-24

URL:

http://hdl.handle.net/2433/159945

\section{RIGHT:}

(C) 2012 Elsevier Inc:; This is not the published version. Please cite only the published version.; この論文は出版社版でありません。引用の際に は出版社版をご確認ご利用ください。 
NMR study of xenotropic murine leukemia virus-related virus protease in a complex with amprenavir

Ayako Furukawa ${ }^{a}$, Hideyasu Okamura ${ }^{a}$, Ryo Morishita ${ }^{b, c}$, Satoko Matsunaga ${ }^{c}$, Naohiro Kobayashi $^{\mathrm{d}}$, Tsutomu Kodaki ${ }^{\mathrm{a}, \mathrm{e}}$, Akifumi Takaori-Kondo ${ }^{\mathrm{f}}$, Akihide Ryo ${ }^{\mathrm{c}}$, Takashi Nagata ${ }^{\mathrm{a}, \mathrm{e}, *}$ and Masato Katahira ${ }^{\text {a,e,g,*** }}$

${ }^{a}$ Institute of Advanced Energy, Kyoto University, Gokasho, Uji, Kyoto 611-0011, Japan

${ }^{\mathrm{b}}$ CellFree Sciences Co., Ltd., Ehime Univ. Venture Business Laboratory, Matsuyama 790 - 8577, Japan

${ }^{c}$ Department of Microbiology, Yokohama City University School of Medicine, 3-9 Fukuura, Kanazawa-ku, Yokohama 236-0004, Japan

${ }^{\mathrm{d}}$ Institute for Protein Research, Osaka University, 3-2 Yamadaoka, Suita, Osaka 565-0871, Japan

${ }^{\mathrm{e}}$ Graduate School of Energy Science, Kyoto University, Gokasho, Uji, Kyoto 611-0011, Japan

${ }^{\mathrm{f}}$ Department of Hematology and Oncology, Graduate School of Medicine, Kyoto University, Sakyo-ku, Kyoto 606-8507, Japan

${ }^{g}$ CREST, JST, 5-3 Yonban-cho, Chiyoda-ku, Tokyo 102-8666, Japan

\section{Keywords}

XMRV, protease, cell-free protein synthesis, NMR

Corresponding authors

E-mail addresses: katahira@iae.kyoto-u.ac.jp (M. Katahira),nagatat@iae.kyoto-u.ac.jp (T. Nagata) 


\begin{abstract}
Xenotropic murine leukemia virus-related virus (XMRV) is a virus created through recombination of two murine leukemia proviruses under artificial conditions during the passage of human prostate cancer cells in athymic nude mice. The homodimeric protease (PR) of XMRV plays a critical role in the production of functional viral proteins and is a prerequisite for viral replication. We synthesized XMRV PR using the wheat germ cell-free expression system and carried out structural analysis of XMRV PR in a complex with an inhibitor, amprenavir (APV), by means of NMR. Five different combinatorially ${ }^{15} \mathrm{~N}$-labeled samples were prepared and backbone resonance assignments were made by applying Otting's method, with which the amino acid types of the $\left[{ }^{1} \mathrm{H},{ }^{15} \mathrm{~N}\right] \mathrm{HSQC}$ resonances were automatically identified using the five samples (Wu et al., 2006). A titration experiment involving APV revealed that one APV molecule binds to one XMRV PR dimer. For many residues, two distinct resonances were observed, which is thought to be due to the structural heterogeneity between the two protomers in the APV:XMRV PR = 1:2 complex. PR residues at the interface with APV have been identified on the basis of chemical shift perturbation and identification of the intermolecular NOEs by means of filtered NOE experiments. Interestingly, chemical shift heterogeneity between the two protomers of XMRV PR has been observed not only at the interface with APV but also in regions apart from the interface. This indicates that the structural heterogeneity induced by the asymmetry of the binding of APV to the XMRV PR dimer is transmitted to distant regions. This is in contrast to the case of the APV:HIV-1 PR complex, in which the structural heterogeneity is only localized at the interface. Long-range transmission of the structural change identified for the XMRV PR complex might be utilized for the discovery of a new type of drug.
\end{abstract}




\section{Introduction}

Xenotropic murine leukemia virus-related virus (XMRV) has been implicated in prostate cancer [1] and chronic fatigue syndrome [2]. These reports have attracted much attention since a causal relationship between a retrovirus and a human disease had only been known for HIV and AIDS [3]. After several years of controversial research, a recent study has indicated that XMRV was generated through the recombination of two proviruses during the passaging of human tumors in mice [4]. Thus, $\mathrm{XMRV}$ is as not yet regarded as an etiological agent for human diseases, however, it can be considered as a rational example of a gammaretrovirus that can infect human cells and replicate within them.

Proteases (PRs) of viruses such as those of the HIV-1 and hepatitis $\mathrm{C}$ ones have been prime targets for antiviral drug development. Although hundreds of inhibitors have been discovered for HIV-1 PR for example, only a few of them are potent antiprotease drugs that are in clinical use [5]. Additionally, long-term administration of these drugs causes the emergence of drug-resistant mutants [6]. It is expected that a detailed description of the interaction between a newly found protease of the retrovirus family and its cognate substrate or inhibitor may provide valuable information.

XMRV PR is a homodimer, each subunit (protomer) comprising 125 amino acids with a single catalytic Asp residue [7,8]. XMRV PR processes viral polyproteins to yield mature proteins required in the viral life cycle, as is the case for HIV-1 PR. The sequence identity between and similarity of these two proteins are $21 \%$ and $27 \%$, respectively, over 99 amino acid residues with a single gap (sequences aligned with ClustalW2 [9,10]). The crystal structures of XMRV PR have been solved in both the free form and complex forms with several inhibitors [7,8]. The fold of XMRV PR is similar to that of HIV-1 PR. The overall structural similarity between the protomer of XMRV PR (PDB ID: 3NR6; [7]) and that of HIV-1 PR (99 amino acids; PDB ID: 3HVP; [11]) in the free form was found to be $1.04 \AA$ of RMSD over $53 \mathrm{C}^{\alpha}$ atoms (calculated using CHIMERA [12]).

In the present study, we used our established wheat germ protein production system to obtain otherwise cell-toxic XMRV PR [13]. This system allowed us to obtain not only a large amount of free and amprenavir (APV)-bound XMRV PR, but also uniformly and combinatorially isotope-labeled samples. The latter isotope-labeled samples made it possible to apply Otting's rapid assignment strategy [14] in combination with conventional protocols [15]. It was critical to utilize this strategy for accomplishment of the assignments. We found that the asymmetry of the binding for the APV:XMRV $\mathrm{PR}=1: 2$ complex results in the structural heterogeneity between the two protomers of XMRV PR at the interface, and, but more importantly, in regions distant from the interface. In the case of the binding of APV to HIV-1 PR, in contrast, the asymmetry causes structural heterogeneity only at the interface.

\section{Materials and methods}

\subsection{Sample preparation}

XMRV PR comprising 1-125 residues with an additional threonine residue at the C-terminal end, was synthesized by means of our wheat germ cell-free expression system as an N-terminal glutathione-S-transferase (GST) fusion protein. The XMRV PR fragment was cloned by PCR from the 
XMRV VP62 clone [16,17] (UniProt ID: A1Z651) and subcloned into pEU-E01-GW [18] with the DNA sequences of the GST gene and Tobacco Etch Virus Protease (TEV) cleavage site. This plasmid DNA was subjected to in vitro transcription and cell-free protein synthesis with the wheat germ protein production system [13]. Cell-free protein production was carried out using the ENDEXT.AN. Wheat Germ Expression Kit and according to the instructions provided by the supplier (CellFree Sciences Co., Ltd., Matsuyama, Japan). The cell-free protein production and cleavage purification were also carried out using an automatic robot, Protemist DTII (CellFree Sciences Co., Ltd.), basically according to manufacturer's instructions. GST-XMRV PR was synthesized either in the absence or presence of APV. After the synthesis, the protein solution was loaded onto a Glutathione-Sepharose 4B column (GE Healthcare), and the bound GST-XMRV PR was washed thoroughly with PBS buffer. Subsequently, GST-fused TEV protease (Nacalai Tesque) was applied to cleave the XMRV PR off on-column. The XMRV PR was then collected and dialyzed against either $20 \mathrm{mM}$ sodium phosphate ( $\mathrm{pH} 6.5$ ), $50 \mathrm{mM} \mathrm{NaCl}$ and $10 \mu \mathrm{M}$ APV for the APV-bound XMRV PR or $20 \mathrm{mM}$ Tris- $\mathrm{HCl}(\mathrm{pH} \mathrm{8.0)}$ and $50 \mathrm{mM} \mathrm{NaCl}$ for APV-free XMRV PR. As substrates for protein synthesis, isotropically labeled amino acids were used to synthesize uniformly ${ }^{15} \mathrm{~N}$ - and ${ }^{15} \mathrm{~N},{ }^{13} \mathrm{C}$-labeled $\left(\left[\mathrm{U}_{-}{ }^{15} \mathrm{~N}\right]\right.$ and $\left[\mathrm{U}_{-}{ }^{13} \mathrm{C}, \mathrm{U}_{-}{ }^{15} \mathrm{~N}\right]$, respectively) XMRV PRs. Five different combinatorially ${ }^{15} \mathrm{~N}$-labeled samples of XMRV PR were prepared following Otting's labeling scheme, as shown in Fig. 1C [14].

\subsection{NMR spectroscopy}

For XMRV PR in a complex with APV, NMR spectra were acquired at $22.9^{\circ} \mathrm{C}$ using Bruker 600 and $950 \mathrm{MHz}$ spectrometers each equipped with a cryoprobe. Data were processed and analyzed using NMRPipe [19] and Kujira [20]. The sample solutions comprised $0.1-0.3 \mathrm{mM}$ protein in a complex with APV, $20 \mathrm{mM}$ sodium phosphate $(\mathrm{pH} 6.5), 50 \mathrm{mM} \mathrm{NaCl}, 10 \mu \mathrm{M}$ APV, and $5 \% \mathrm{D}_{2} \mathrm{O}$. The amino acid in $\left[{ }^{1} \mathrm{H},{ }^{15} \mathrm{~N}\right]$ HSQC spectra were identified using the five different combinatorially ${ }^{15} \mathrm{~N}$-labeled samples according to $\mathrm{Wu}$ et al. [14]. Sequential assignments of the main chain resonances were made by means of conventional triple resonance experiments [15,21,22]. The main chain ${ }^{1} \mathrm{H}^{\mathrm{N}}$ and ${ }^{15} \mathrm{~N}$ chemical shift heterogeneity between the two protomers in the APV:XMRV PR=1:2 complex was defined as $\left\{\left(\Delta \delta^{1} \mathrm{H}^{\mathrm{N}}\right)^{2}+\left(\Delta \delta^{15} \mathrm{~N} / 5\right)^{2}\right\}^{1 / 2}$, where $\Delta \delta^{1} \mathrm{H}^{\mathrm{N}}$ and $\Delta \delta^{15} \mathrm{~N}$ are the chemical shift differences between the two protomers for ${ }^{1} \mathrm{H}^{\mathrm{N}}$ and ${ }^{15} \mathrm{~N}$ resonances, respectively. The secondary structures of XMRV PR in a complex with APV was identified based on ${ }^{13} \mathrm{C}^{\alpha},{ }^{13} \mathrm{C}^{\beta}$ and ${ }^{13} \mathrm{C}^{\prime}$ chemical shift values [23]. The intermolecular NOEs between XMRV PR and APV were obtained from $2 \mathrm{D}[\mathrm{F} 1, \mathrm{~F} 2]{ }^{13} \mathrm{C}$, ${ }^{15} \mathrm{~N}$-filtered NOESY (120 ms mixing time), 2D [F2] ${ }^{13} \mathrm{C},{ }^{15} \mathrm{~N}$-filtered NOESY (120 ms mixing time), $3 \mathrm{D}[\mathrm{F} 1]{ }^{13} \mathrm{C},{ }^{15} \mathrm{~N}$-filtered, [F2] ${ }^{13} \mathrm{C}$-edited NOESY (120 ms mixing time) [24], and 3D ${ }^{13} \mathrm{C}$-edited NOESY (78 ms mixing time) spectra.

For free XMRV PR, NMR spectra were acquired at $15.0^{\circ} \mathrm{C}$ using a Bruker $600 \mathrm{MHz}$ spectrometer equipped with a cryoprobe. The sample solutions comprised $\sim 0.1 \mathrm{mM}$ protein, $20 \mathrm{mM}$ Tris- $\mathrm{HCl}(\mathrm{pH}$ 8.0), $50 \mathrm{mM} \mathrm{NaCl}$ and $5 \% \mathrm{D}_{2} \mathrm{O}$. Partial assignments of the main chain ${ }^{1} \mathrm{H}^{\mathrm{N}}$ and ${ }^{15} \mathrm{~N}$ resonances were made using 3D ${ }^{15} \mathrm{~N}$-edited NOESY spectra utilizing on the assignments for the XMRV PR-APV 
complex as a reference. The main chain ${ }^{1} \mathrm{H}^{\mathrm{N}}$ and ${ }^{15} \mathrm{~N}$ chemical shift perturbations between the free and APV-bound forms of XMRV PR were defined as $\left\{\left(\Delta \delta^{1} \mathrm{H}^{\mathrm{N}}\right)^{2}+\left(\Delta \delta^{15} \mathrm{~N} / 5\right)^{2}\right\}^{1 / 2}$, where $\Delta \delta^{1} \mathrm{H}^{\mathrm{N}}$ and $\Delta \delta^{15} \mathrm{~N}$ are the chemical shift differences between the free and APV-bound forms for ${ }^{1} \mathrm{H}^{\mathrm{N}}$ and ${ }^{15} \mathrm{~N}$ resonances, respectively. For the APV-bound form, the average of the chemical shift values for the two protomers was used to calculate the differences.

\section{Results and Discussion}

\subsection{Characteristics of the $\left[{ }^{1} H,{ }^{15} \mathrm{~N}\right] \mathrm{HSQC}$ spectrum of the XMRV PR in complex with APV}

We have successfully obtained cell-toxic XMRV PR by using a cell-free protein synthesis system in both the presence and absence of APV. Moreover, we were able to prepare $\left[\mathrm{U}-{ }^{15} \mathrm{~N}\right]$ and $\left[\mathrm{U}-{ }^{13} \mathrm{C}, \mathrm{U}-{ }^{15} \mathrm{~N}\right]$ labeled, and five different combinatorially ${ }^{15} \mathrm{~N}$-labeled samples of XMRV PR, all of which critically facilitated our NMR analyses.

First, titration with APV was carried out. Disappearance of the resonances of XMRV PR in the free state and simultaneous appearance of new resonances for the APV-bound form were observed in the $\left[{ }^{1} \mathrm{H},{ }^{15} \mathrm{~N}\right]$ HSQC spectrum during the titration. This indicates that the free and bound forms of XMRV PR are in a slow exchange regime on an NMR time scale. Such spectral changes for XMRV PR were seen until APV:XMRV PR=1:2, but no further change was seen on further addition of APV. This indicates that the stoichiometry is one APV molecule per one XMRV PR dimer (two XMRV PR monomers).

Each protomer comprises 126 amino acid residues, including 12 proline ones. If the homodimer exhibits symmetry, maximally 113 backbone resonances are expected to be observed, because those of the N-terminus and proline residues do not appear. However, the $\left[{ }^{1} \mathrm{H},{ }^{15} \mathrm{~N}\right] \mathrm{HSQC}$ spectrum contained more than 1.5-times as many backbone resonances as expected (Fig. 1A). This observation reveals that the APV-bound XMRV PR exhibits asymmetry.

\subsection{Sequence-specific resonance assignments and identification of the secondary structure of the} $X M R V P R$ in a complex with APV

We applied Otting's strategy, with which amino acids could be automatically identified on the basis of the comparison of five spectra [14]. Assignments of the ${ }^{1} \mathrm{H}^{\mathrm{N}},{ }^{15} \mathrm{~N},{ }^{13} \mathrm{C}^{\alpha},{ }^{13} \mathrm{C}^{\beta}$, and ${ }^{13} \mathrm{C}^{\prime}$ backbone resonances of XMRV PR in complex with APV were carried out with the knowledge of the amino acid. Almost all of the observed resonances were successfully assigned. It was found that two distinct backbone resonances were observed for many residues of XMRV PR in a complex with APV, which resulted in more than 1.5 times as many backbone resonances as expected. The observation of two distinct peaks for many residues must be due to that the two protomers of the XMRV PR dimer are not identical to each other in the APV:XMRV PR = 1:2 complex.

The secondary structure of the XMRV PR in a complex with APV was identified according to the ${ }^{13} \mathrm{C}^{\alpha},{ }^{13} \mathrm{C}^{\beta}$ and ${ }^{13} \mathrm{C}^{\prime}$ chemical shift values [23] (Fig. 1D). It was noticed that the two protomers exhibited almost identical secondary structures. The solution structure of XMRV PR in a complex with APV 
turned out to be basically the same as the crystal structure, which was solved recently [8], as shown in Fig. 1D.

\subsection{Binding mode of APV as to XMRM PR in solution}

The quality of the $\left[{ }^{1} \mathrm{H},{ }^{15} \mathrm{~N}\right]$ HSQC spectrum of APV-free XMRV PR appeared to be rather poor due to line broadening of resonances in comparison with in the case of APV-bound XMRV PR. By comparing the spectral patterns of the $3 \mathrm{D}{ }^{15} \mathrm{~N}$-edited NOESY spectra between the APV-bound and APV-free forms of XMRV PR, we achieved partial assignment of the main chain ${ }^{1} \mathrm{H}^{\mathrm{N}}$ and ${ }^{15} \mathrm{~N}$ resonances of APV-free XMRV PR. It was found on this analysis that only one set of resonances was observed for APV-free XMRV PR. This indicates that the two protomers are symmetric as to each other in the APV-free form. We then calculated the chemical shift perturbations of the backbone resonances of XMRV PR upon APV binding, and mapped them onto the crystal structure (Fig. 2A). The largest perturbations were observed for the residues located close to APV in the crystal structure (Fig. 2A). This strongly suggests that in solution APV resides at a similar position to as observed in the crystal.

In order to further confirm the interface of XMRV PR with APV, identification of intermolecular NOEs was attempted. First, the ${ }^{1} \mathrm{H}^{\mathrm{N}}$ resonances of APV bound to XMRV PR were assigned using the $2 \mathrm{D}[\mathrm{F} 1, \mathrm{~F} 2]{ }^{13} \mathrm{C},{ }^{15} \mathrm{~N}$-filtered NOESY spectrum (Fig. 2B). Secondly, the intermolecular NOEs between XMRV PR and APV were discriminated by comparing the $2 \mathrm{D}[\mathrm{F} 1, \mathrm{~F} 2]{ }^{13} \mathrm{C},{ }^{15} \mathrm{~N}$-filtered NOESY and $2 \mathrm{D}[\mathrm{F} 2]{ }^{13} \mathrm{C},{ }^{15} \mathrm{~N}$-filtered NOESY spectra (Fig. 2B). That is, NOEs observed in the latter spectrum but not in the former one were judged to be intermolecular NOEs. Finally, these intermolecular NOEs were assigned by combinational use of the $3 \mathrm{D}[\mathrm{F} 1]{ }^{13} \mathrm{C},{ }^{15} \mathrm{~N}$-filtered, [F2] ${ }^{13} \mathrm{C}$-edited NOESY, 3D

${ }^{13} \mathrm{C}$-edited NOESY, and 3D ${ }^{15} \mathrm{~N}$-edited NOESY spectra. Consequently, we were able to identify and assign the intermolecular NOEs between APV (P1, P1', P2 and P2'), and either the methyl groups of A35, V39, V54, A57, L83 and L92 or the aromatic ring of Y90 (Fig. 2B). These intermolecular NOEs turned out to be fully consistent with the crystal structure of the APV:XMRV PR complex. All of the listed amino acid residues are indeed located close to APV in the crystal structure (Fig. 2C). This revealed that in solution APV binds to XMRV in the same manner as observed in the crystal.

\subsection{Long-range transmission of the structural heterogeneity between two protomers in the APV:XMRV} PR complex

The ${ }^{1} \mathrm{H}^{\mathrm{N}},{ }^{15} \mathrm{~N}$ chemical shift heterogeneity of the backbone resonances between the two protomers of XMRV PR in a complex with APV was calculated and mapped onto the crystal structure of the XMRV PR:APV complex (Fig. 3A). It turned out that chemical shift heterogeneity was observed not only at the APV-binding site (D32, A35, Q36 and L92), but also at positions distant from the APV-binding site (S38, L73, H80, S81, L97 and L122). This suggests that the structural heterogeneity occurs not only at the interface with APV but also in these regions in the APV:XMRV PR $=1: 2$ complex.

Then, we examined the structural heterogeneity of the crystal structure of the APV:XMRV PR 
complex. The backbone atoms of the two protomers were superimposed and the differences in position between the $\mathrm{C}^{\alpha}$ atoms of the two protomers were calculated to estimate the structural heterogeneity in the crystal. The $\mathrm{C}^{\alpha}-\mathrm{C}^{\alpha}$ distance was mapped on the crystal structure of the APV:XMRV PR complex (Fig. 3B). It is found that structural heterogeneity is present not only at the interface with APV but also in regions distant in the crystal structure too. Thus, it was found that the structural heterogeneity exhibits long-range transmission in the APV:XMRV PR=1:2 complex in both solution and crystal states.

3.5 Long-range transmission of the structural heterogeneity is specific to the APV:XMRV PR complex, not being observed in the APV:HIV-1 PR complex

Next, we examined if the long-range transmission of the structural heterogeneity occurs for PR of another virus, HIV-1. The ${ }^{1} \mathrm{H}^{\mathrm{N}},{ }^{15} \mathrm{~N}$ chemical shift heterogeneity between the two protomers of HIV-1 (D25N) PR in a complex with a peptide substrate was calculated using the previously reported data [25]. Here, heterogeneity in solution was analyzed for the peptide-bound form, because NMR data on HIV-1 in a complex with APV are not available. It has been confirmed that the mutant (D25N) and wild-type HIV-1 PRs exhibited essentially identical backbone chemical shift values, except for those of mutated residue 25 and adjacent residues [25]. Since the peptide substrate is larger than APV in size and has a highly asymmetric structure, this system could be used instead to compare the heterogeneity between the XMRV and HIV-1 PRs. The chemical shift heterogeneity values were mapped onto the crystal structure of the HIV-1 PR (D25N) in a complex with APV (Fig. 3C). The chemical shift heterogeneity was mostly observed for residues located close the substrate/inhibitor-binding site. This indicates that the structural heterogeneity is localized around the substrate binding site and that the structural heterogeneity is not transmitted to distant regions in the HIV-1 PR, which is in contrast to the case of the XMRV PR.

In order to further confirm the localization of the structural heterogeneity for HIV-1 PR, the crystal structure of the APV:HIV-1 PR (D25N) complex was examined. The differences in the positions of the $\mathrm{C}^{\alpha}$ atoms of the two protomers were calculated and mapped onto the crystal structure (Fig. 3D). The structural heterogeneity was mostly observed for the residues that are close to the substrate-binding site. This is consistent with the conclusion derived from NMR data in solution for HIV-1 PR. Thus, the long-range transmission of the structural heterogeneity does not occur in HIV-1 $\mathrm{PR}$, but is specific to XMRV-PR.

3.6 Implications for discovery of new types of drug on the basis of the identification of long-range transmission of the structural heterogeneity

It has been pointed out for HIV-1 PR that opening and closing of the flap of the binding site results in compression and expansion of the shallow groove indicated by the arrow in Fig. 3D, respectively [26,27]. Recently, the peripheral surface of HIV-1 PR, which is relatively apart from the binding site, was suggested to be a good target for the discovery of allosteric inhibitors. A 
fragment-based screen against HIV-1 PR in a complex with TL-3, a universal inhibitor of retroviral PRs, has been employed using the Active Sight fragment library and X-ray crystallography [28]. The authors of that study discovered that 2-methylcyclohexanol binds to the 'exo site' just by the shallow groove [28]. This compound binds to the shallow groove and restricts its compression, and thus prevents the flap from opening. Therefore, it was proposed that the compound can be used as a starting molecule for the development of high-affinity allosteric inhibitors [28]. This example illustrates that a drug that binds to a position distant from the binding site can affect the binding affinity, being a candidate inhibitor. We have shown that the structural heterogeneity is transmitted away in XMRV PR. This means that the heterogeneous structural changes on binding of APV are transmitted to distant regions. This implies conversely that a drug that binds to a distant site may affect the structure of the substrate-binding site and thus inhibit binding of a substrate. This new type of a drug might be discovered more easily for XMRV PR than for HIV-1 PR. Then, in some cases, the discovered drug might bind to only one protomer of the dimer due to structural heterogeneity, as is the case for 2-methylcyclohexanol [28]. 


\section{Acknowledgements}

This work was supported by grants from MEXT (23570146, 23657072, 24121714, and 24113710), JST-SENTAN, JST-CREST, the Sumitomo-Denko Foundation and the Iwatani Foundation.

\section{References}

[1] R. Schlaberg, D.J. Choe, K.R. Brown, H.M. Thaker, I.R. Singh, XMRV is present in malignant prostatic epithelium and is associated with prostate cancer, especially high-grade tumors, Proc Natl Acad Sci U S A 106 (2009) 16351-16356.

[2] V.C. Lombardi, F.W. Ruscetti, J. Das Gupta, M.A. Pfost, K.S. Hagen, D.L. Peterson, S.K. Ruscetti, R.K. Bagni, C. Petrow-Sadowski, B. Gold, M. Dean, R.H. Silverman, J.A. Mikovits, Detection of an infectious retrovirus, XMRV, in blood cells of patients with chronic fatigue syndrome, Science 326 (2009) 585-589.

[3] S. Broder, R.C. Gallo, A pathogenic retrovirus (HTLV-III) linked to AIDS, N Engl J Med 311 (1984) 1292-1297.

[4] T. Paprotka, K.A. Delviks-Frankenberry, O. Cingoz, A. Martinez, H.J. Kung, C.G. Tepper, W.S. Hu, M.J. Fivash, Jr., J.M. Coffin, V.K. Pathak, Recombinant origin of the retrovirus XMRV, Science 333 (2011) 97-101.

[5] I.R. Singh, J.E. Gorzynski, D. Drobysheva, L. Bassit, R.F. Schinazi, Raltegravir is a potent inhibitor of XMRV, a virus implicated in prostate cancer and chronic fatigue syndrome, PLoS One 5 (2010) e9948.

[6] E.J. Arts, D.J. Hazuda, HIV-1 Antiretroviral Drug Therapy, Cold Spring Harb Perspect Med 2 (2012) a007161.

[7] M. Li, F. Dimaio, D. Zhou, A. Gustchina, J. Lubkowski, Z. Dauter, D. Baker, A. Wlodawer, Crystal structure of XMRV protease differs from the structures of other retropepsins, Nat Struct Mol Biol 18 (2011) 227-229.

[8] M. Li, A. Gustchina, K. Matuz, J. Tozser, S. Namwong, N.E. Goldfarb, B.M. Dunn, A. Wlodawer, Structural and biochemical characterization of the inhibitor complexes of xenotropic murine leukemia virus-related virus protease, FEBS J 278 (2011) 4413-4424.

[9] M. Goujon, H. McWilliam, W. Li, F. Valentin, S. Squizzato, J. Paern, R. Lopez, A new bioinformatics analysis tools framework at EMBL-EBI, Nucleic Acids Res 38 (2010) W695-699.

[10] M.A. Larkin, G. Blackshields, N.P. Brown, R. Chenna, P.A. McGettigan, H. McWilliam, F. Valentin, I.M. Wallace, A. Wilm, R. Lopez, J.D. Thompson, T.J. Gibson, D.G. Higgins, Clustal W and Clustal X version 2.0, Bioinformatics 23 (2007) 2947-2948.

[11] A. Wlodawer, M. Miller, M. Jaskolski, B.K. Sathyanarayana, E. Baldwin, I.T. Weber, L.M. Selk, L. Clawson, J. Schneider, S.B. Kent, Conserved folding in retroviral proteases: crystal structure of a synthetic HIV-1 protease, Science 245 (1989) 616-621.

[12] E.F. Pettersen, T.D. Goddard, C.C. Huang, G.S. Couch, D.M. Greenblatt, E.C. Meng, T.E. Ferrin, 
UCSF Chimera--a visualization system for exploratory research and analysis, J Comput Chem 25 (2004) 1605-1612.

[13] S. Matsunaga, T. Sawasaki, H. Ode, R. Morishita, A. Furukawa, R. Sakuma, W. Sugiura, H. Sato, M. Katahira, A. Takaori-Kondo, N. Yamamoto, A. Ryo, Molecular and enzymatic characterization of XMRV protease by a cell-free proteolytic analysis, J Proteomics (2012).

[14] P.S. Wu, K. Ozawa, S. Jergic, X.C. Su, N.E. Dixon, G. Otting, Amino-acid type identification in 15N-HSQC spectra by combinatorial selective 15N-labelling, J Biomol NMR 34 (2006) $13-21$.

[15] J. Cavanagh, W.J. Fairbrother, A.G. Palmer, N.J. Skelton, M. Rance, Protein NMR Spectroscopy: Principles and Practice, Second ed., Academic Press, San Diego, CA, 2007.

[16] R. Sakuma, T. Sakuma, S. Ohmine, R.H. Silverman, Y. Ikeda, Xenotropic murine leukemia virus-related virus is susceptible to AZT, Virology 397 (2010) 1-6.

[17] A. Urisman, R.J. Molinaro, N. Fischer, S.J. Plummer, G. Casey, E.A. Klein, K. Malathi, C. Magi-Galluzzi, R.R. Tubbs, D. Ganem, R.H. Silverman, J.L. DeRisi, Identification of a novel Gammaretrovirus in prostate tumors of patients homozygous for R462Q RNASEL variant, PLoS Pathog 2 (2006) e25.

[18] H. Takahashi, A. Nozawa, M. Seki, K. Shinozaki, Y. Endo, T. Sawasaki, A simple and high-sensitivity method for analysis of ubiquitination and polyubiquitination based on wheat cell-free protein synthesis, Bmc Plant Biology 9 (2009).

[19] F. Delaglio, S. Grzesiek, G.W. Vuister, G. Zhu, J. Pfeifer, A. Bax, NMRPipe: a multidimensional spectral processing system based on UNIX pipes, J Biomol NMR 6 (1995) 277-293.

[20] N. Kobayashi, J. Iwahara, S. Koshiba, T. Tomizawa, N. Tochio, P. Guntert, T. Kigawa, S. Yokoyama, KUJIRA, a package of integrated modules for systematic and interactive analysis of NMR data directed to high-throughput NMR structure studies, J Biomol NMR 39 (2007) $31-52$.

[21] A. Furukawa, T. Nagata, A. Matsugami, Y. Habu, R. Sugiyama, F. Hayashi, N. Kobayashi, S. Yokoyama, H. Takaku, M. Katahira, Structure, interaction and real-time monitoring of the enzymatic reaction of wild-type APOBEC3G, EMBO J 28 (2009) 440-451.

[22] T. Nagata, E. Niyada, N. Fujimoto, Y. Nagasaki, K. Noto, Y. Miyanoiri, J. Murata, K. Hiratsuka, M. Katahira, Solution structures of the trihelix DNA-binding domains of the wild-type and a phosphomimetic mutant of Arabidopsis GT-1: mechanism for an increase in DNA-binding affinity through phosphorylation, Proteins 78 (2010) 3033-3047.

[23] D.S. Wishart, B.D. Sykes, The 13C chemical-shift index: a simple method for the identification of protein secondary structure using 13C chemical-shift data, J Biomol NMR 4 (1994) 171-180.

[24] C. Zwahlen, P. Legault, S.J.F. Vincent, J. Greenblatt, R. Konrat, L.E. Kay, Methods for measurement of intermolecular NOEs by multinuclear NMR spectroscopy: Application to a bacteriophage lambda N-peptide/boxB RNA complex, J Am Chem Soc 119 (1997) 6711-6721.

[25] E. Katoh, J.M. Louis, T. Yamazaki, A.M. Gronenborn, D.A. Torchia, R. Ishima, A solution NMR 
study of the binding kinetics and the internal dynamics of an HIV-1 protease-substrate complex, Protein Sci 12 (2003) 1376-1385.

[26] V. Hornak, A. Okur, R.C. Rizzo, C. Simmerling, HIV-1 protease flaps spontaneously open and reclose in molecular dynamics simulations, Proc Natl Acad Sci U S A 103 (2006) 915-920.

[27] A.L. Perryman, J.H. Lin, J.A. McCammon, Restrained molecular dynamics simulations of HIV-1 protease: the first step in validating a new target for drug design, Biopolymers 82 (2006) 272-284.

[28] A.L. Perryman, Q. Zhang, H.H. Soutter, R. Rosenfeld, D.E. McRee, A.J. Olson, J.E. Elder, C.D. Stout, Fragment-based screen against HIV protease, Chem Biol Drug Des 75 (2010) 257-268. 


\section{Figure legends}

Figure 1. (A) $\left[{ }^{1} \mathrm{H},{ }^{15} \mathrm{~N}\right]$ HSQC spectrum of uniformly ${ }^{13} \mathrm{C},{ }^{15} \mathrm{~N}$-labeld XMRV PR in a complex with APV. (B) Superposition of the $\left[{ }^{1} \mathrm{H},{ }^{15} \mathrm{~N}\right]$ HSQC spectra of five different combinatorially ${ }^{15} \mathrm{~N}$-labeled XMRV PRs (1-5 of (C)) in a complex with APV, colored red, cyan, green, magenta and blue, respectively. (C) Five different combinatorial labeling schemes [14]. (D) Comparison of the secondary structures of XMRV PR in a complex with APV between the crystal [8] and solution (this work) states.

Figure 2. (A) Mapping of the ${ }^{1} \mathrm{H}^{\mathrm{N}},{ }^{15} \mathrm{~N}$ chemical shift perturbations between the APV-free and APV-bound forms of XMRV PR on to the crystal structure of the APV:XMRV PR complex (PDB accession number 3SM2). The $\mathrm{N}$ - and C-termini of the two protomers are denoted by $\mathrm{N}$ and $\mathrm{C}$, and $\mathrm{N}$ ' and $\mathrm{C}^{\prime}$, respectively. (B) 2D [F2] ${ }^{13} \mathrm{C},{ }^{15} \mathrm{~N}$-filtered NOESY (120 ms mixing time) (blue) and 2D [F1, F2] ${ }^{13} \mathrm{C}$, ${ }^{15} \mathrm{~N}$-filtered NOESY (120 ms mixing time) (red) of XMRV PR in a complex with APV. The assigned intermolecular NOE peaks for XMRV PR and APV are labeled. (C) APV bound to XMRV PR in the crystal (PDB accession number 3SM2). The protomers of XMRV PR are shown in yellow and gray, respectively. APV, is colored magenta. (D) Chemical structure of APV.

Figure 3. (A) Mapping of the ${ }^{1} \mathrm{H}^{\mathrm{N}},{ }^{15} \mathrm{~N}$ chemical shift heterogeneity between the two protomers of XMRV PR in a complex with APV on to the crystal structure of the XMRV PR-APV complex (PDB accession number 3SM2). (B) Mapping of the $C^{\alpha}$ to $C^{\alpha}$ distance between the two superimposed protomers of the XMRV PR-APV crystal structure. The shallow groove is indicated by an arrow. (C) Mapping of the ${ }^{1} \mathrm{H}^{\mathrm{N}},{ }^{15} \mathrm{~N}$ chemical shift heterogeneity between the two protomers of the HIV PR (D25N) in a complex with a peptide substrate [25] on to the crystal structure of the HIV PR (D25N)-APV complex. The structure of APV has been deleted. (D) Mapping of the $\mathrm{C}^{\alpha}$ to $\mathrm{C}^{\alpha}$ distance between the two superimposed protomers in the HIV PR (D25N)-APV crystal structure. The shallow groove is indicated by an arrow. The flap is also indicated. 
Figure 1.

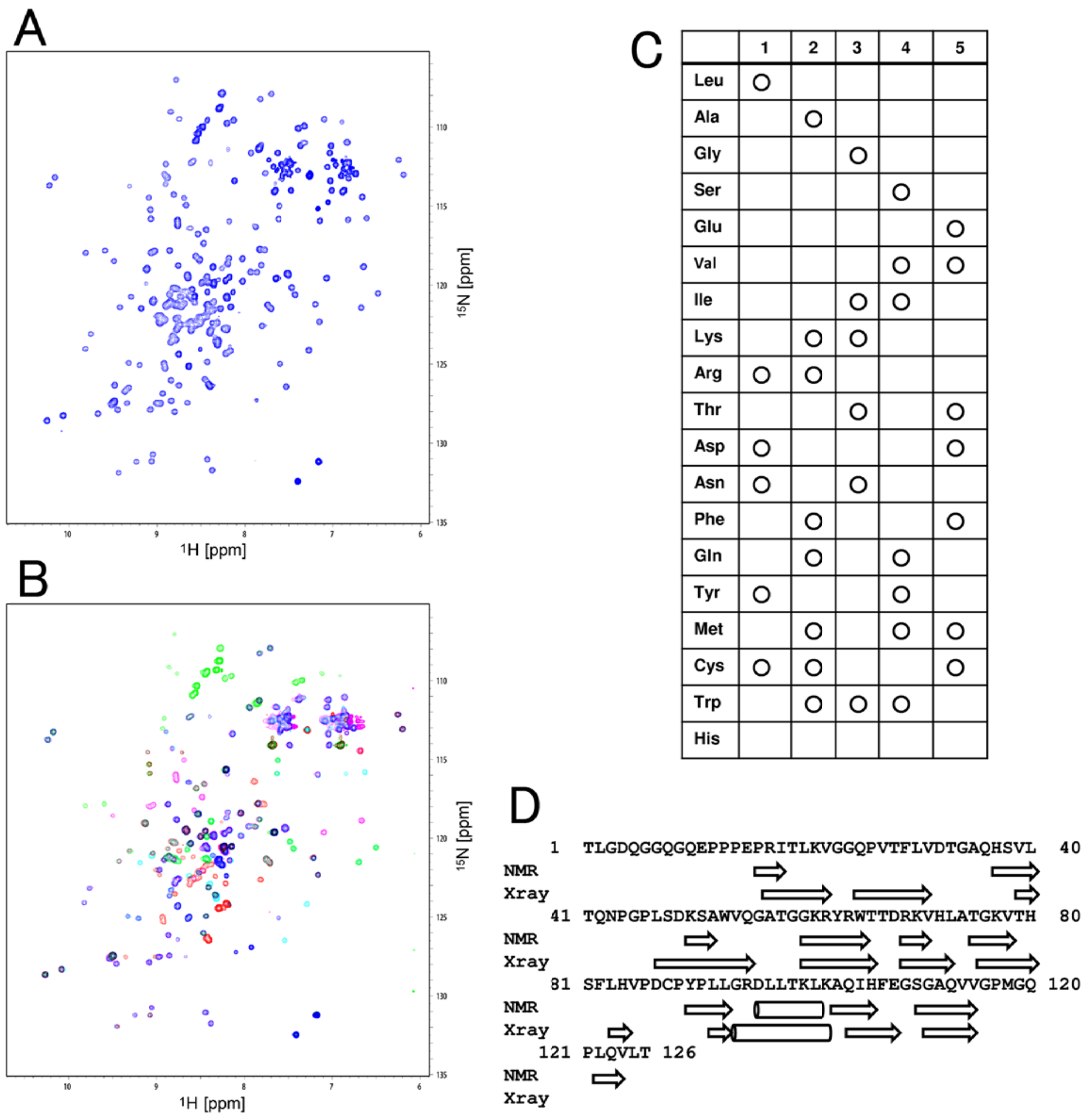


Figure 2.
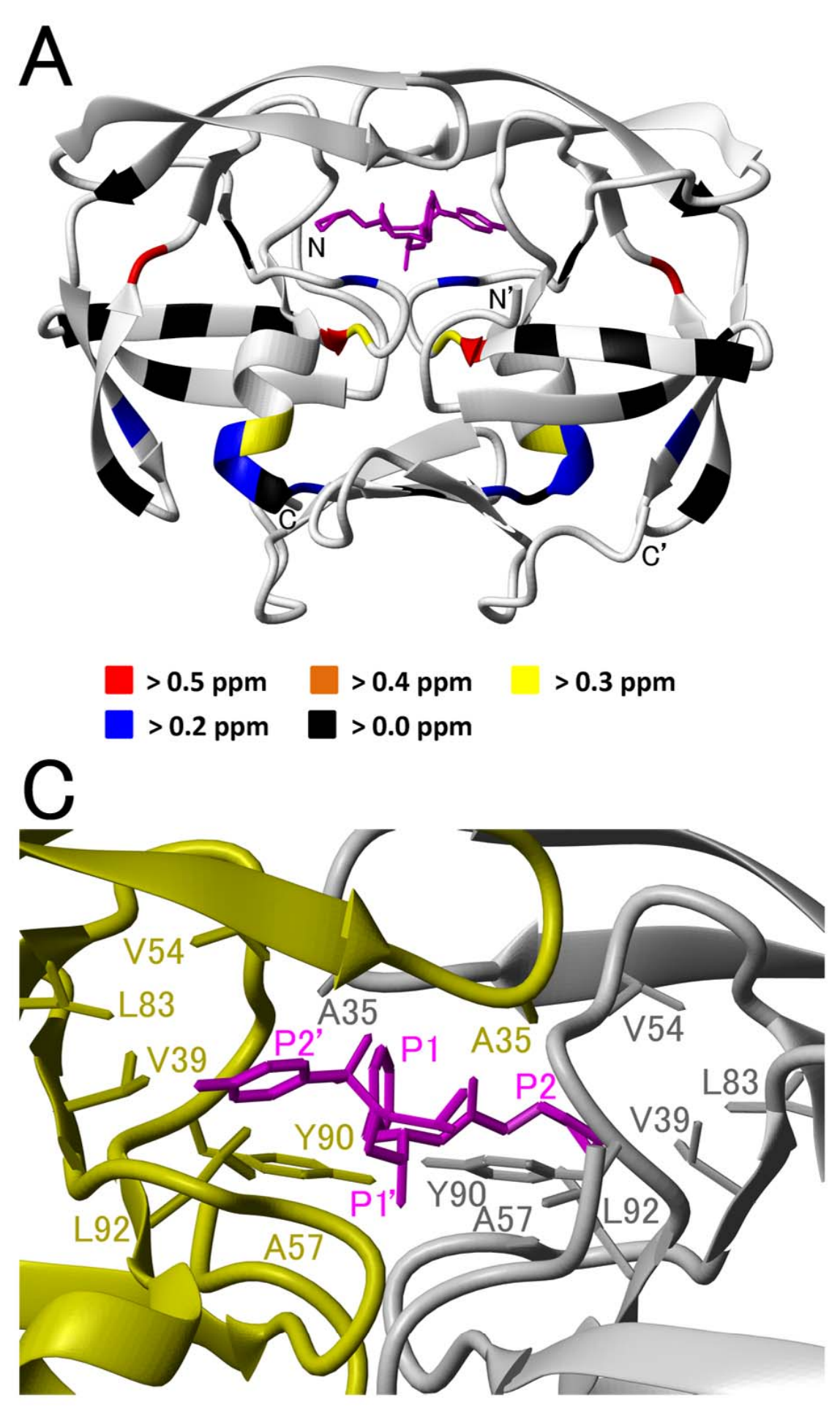

B

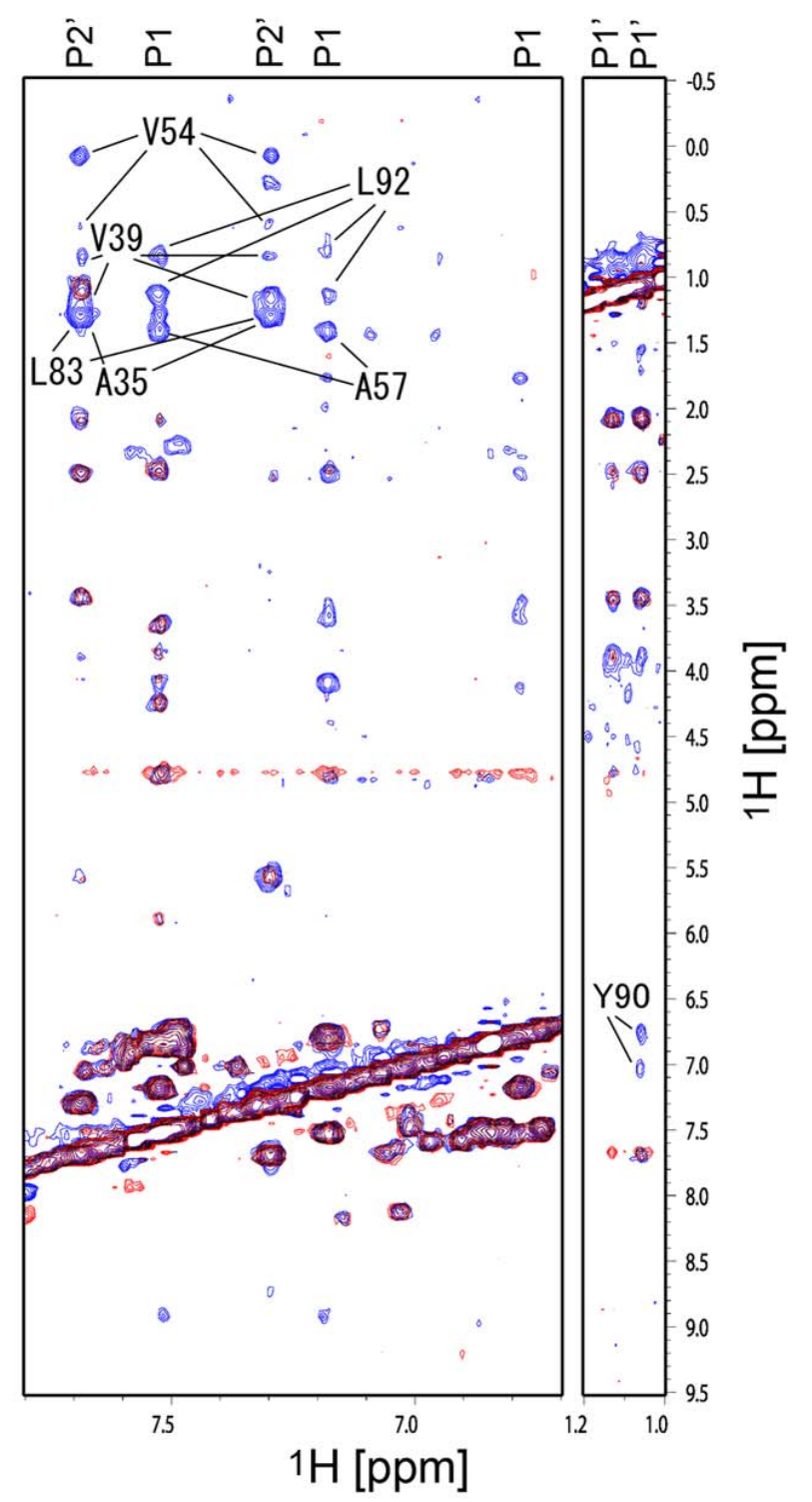

D

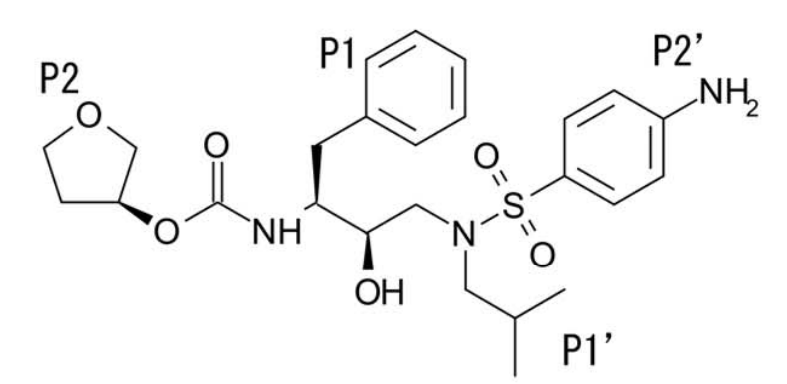


Figure 3.

A

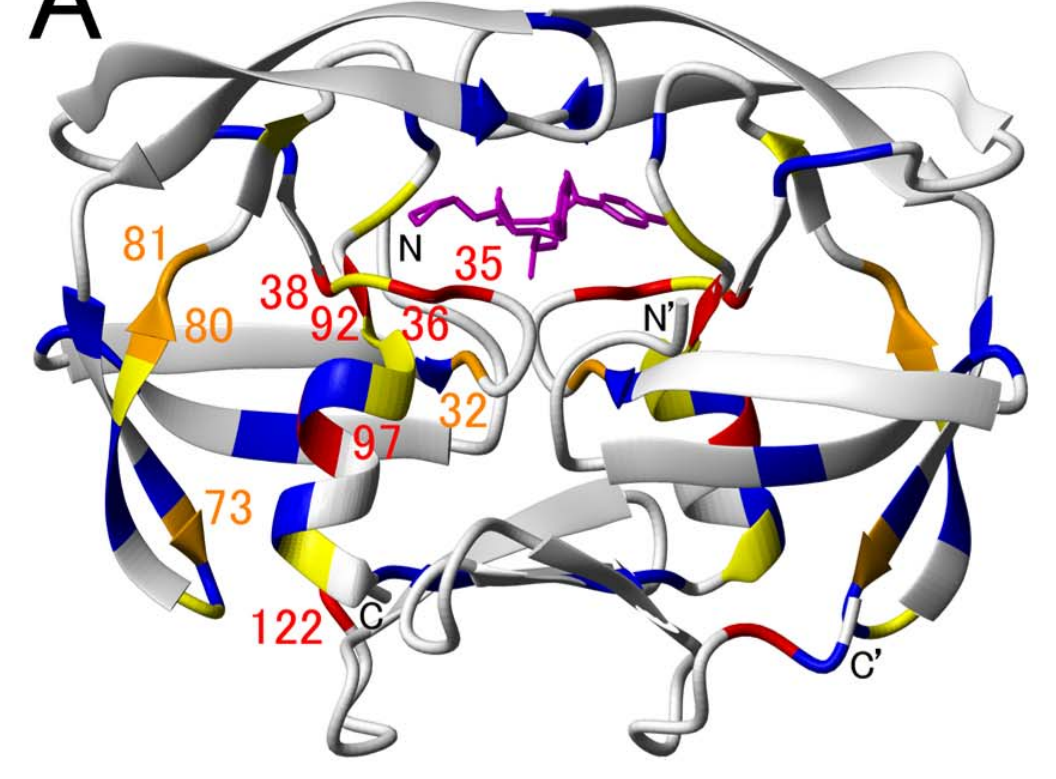

$>0.5 \mathrm{ppm} \square 0.4 \mathrm{ppm}$ $>0.3 \mathrm{ppm} \square 0.2 \mathrm{ppm}$

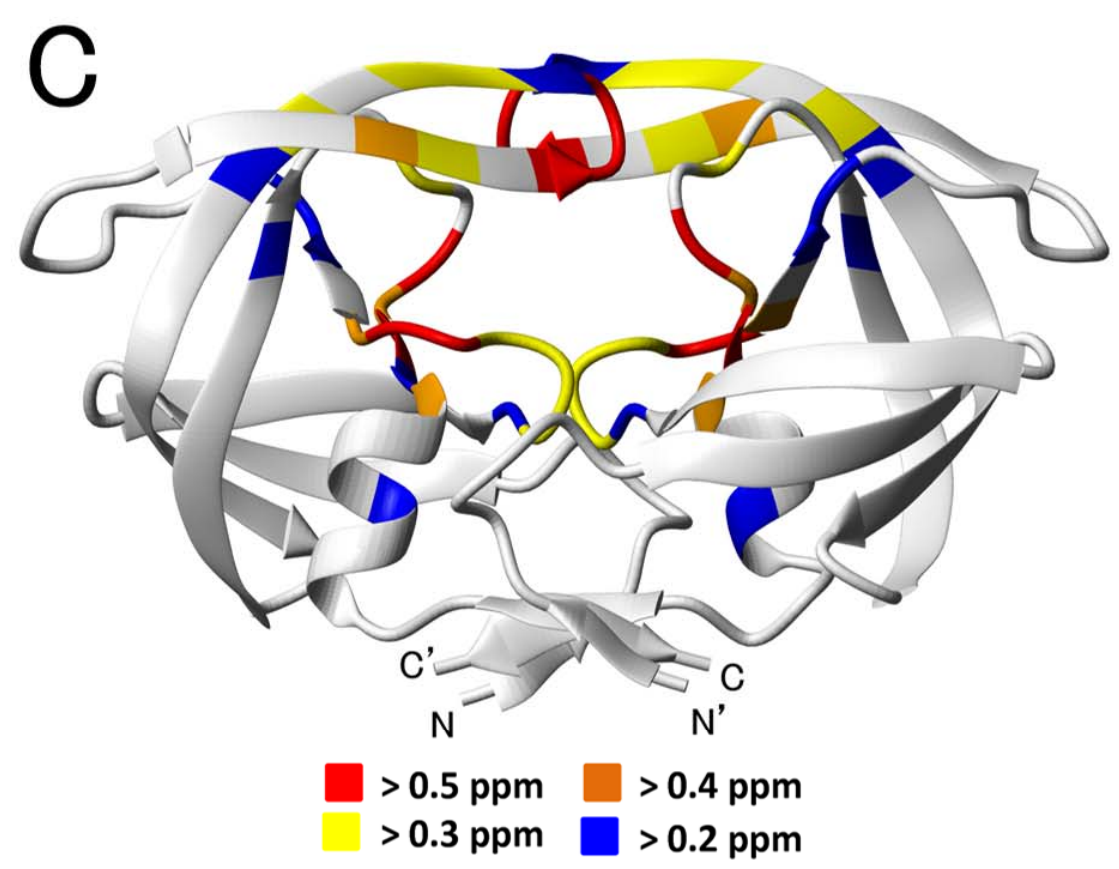

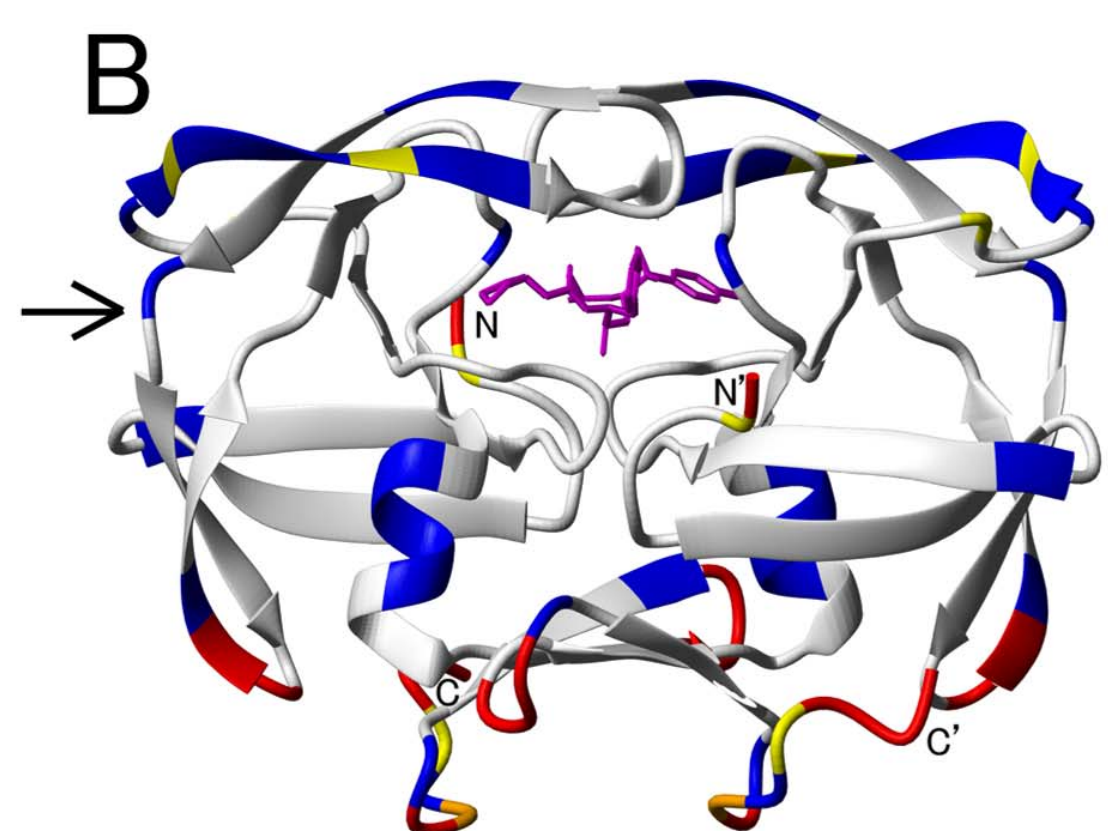

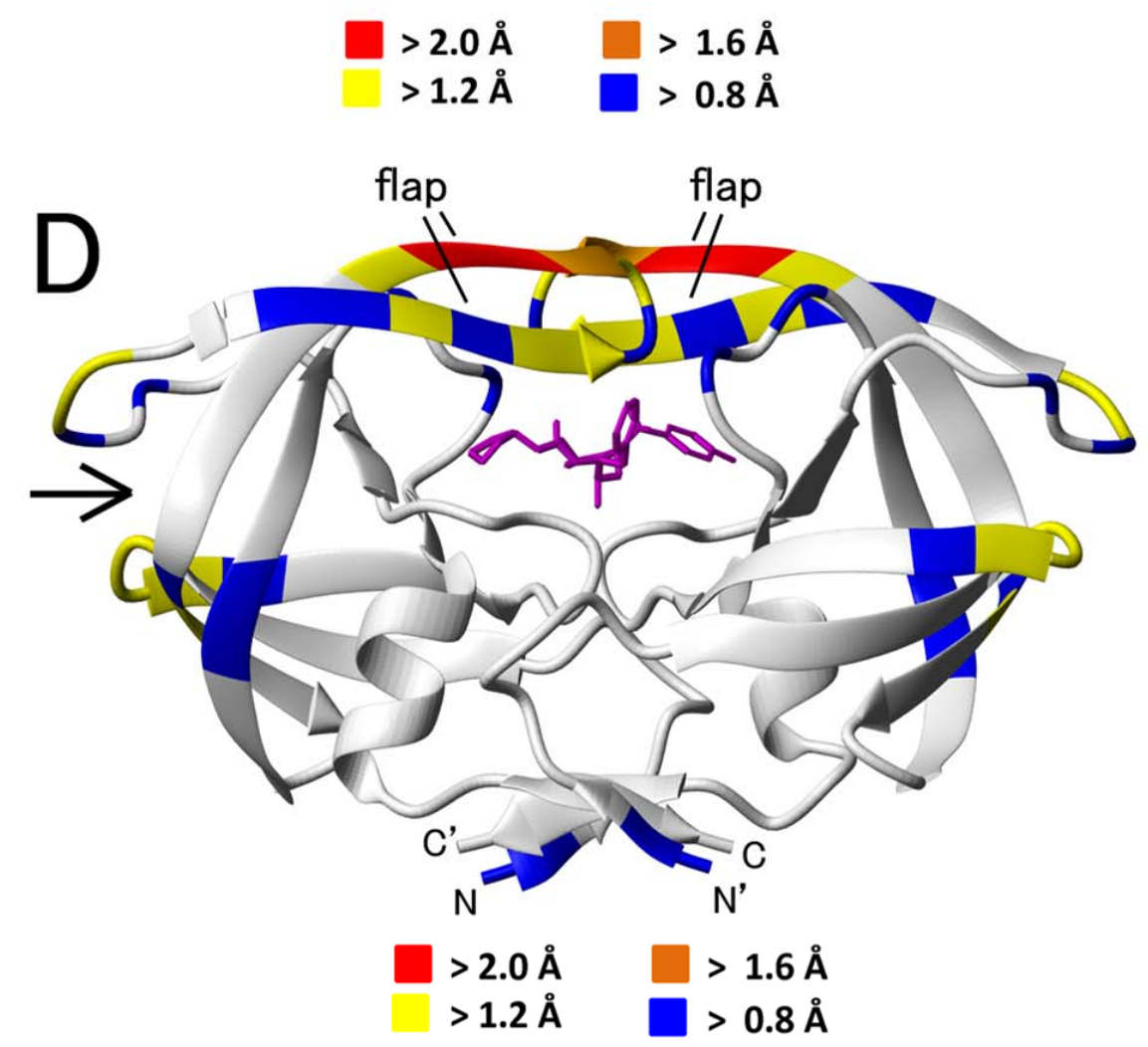

